\title{
Le rapport à la grammaire et à la didactique de la grammaire de futurs enseignants de français langue première au secondaire : un aperçu
}

\author{
Gauvin, Isabelle \\ Université du Québec à Montréal \\ gauvin.isabelle@uqam.ca \\ Aubertin, Philippe \\ Université du Québec à Montréal \\ aubertin.philippe@uqam.ca
}

\section{Introduction}

Lors du symposium La formation initiale des enseignants de français en grammaire: quelles finalités, quelles orientations, quels objectifs ? ${ }^{1}$, les chercheurs français, suisses, belges et québécois réunis ont convenu de l'importance d'intervenir sur les représentations grammaticales des futurs enseignants de français dans le cadre de la formation à l'enseignement. Or, à notre connaissance, aucune recherche récente n'a fait le portrait de ces représentations ${ }^{2}$; nos travaux en cours $^{3}$ ont donc pour objectif de décrire les rapports à la grammaire et les rapports à la didactique de la grammaire qu'entretiennent des étudiants québécois en enseignement du français, langue première ${ }^{4}$.

Cet article vise à discuter en quoi la notion de rapport $\grave{a}$, particulièrement féconde dans les recherches en didactique du français depuis la dernière décennie, peut être importée aux objets "grammaire » et « didactique de la grammaire». Pour y parvenir, nous préciserons d'abord ce que nous entendons par rapport à la grammaire et rapport à la didactique de la grammaire (section 2). Nous fournirons ensuite quelques indications sur la méthode développée pour établir le portrait du rapport à la grammaire et à sa didactique de futurs enseignants de français du secondaire (section 3). Des résultats préliminaires de la recherche seront présentés (section 4) et discutés selon les dimensions du rapport à la grammaire qui émergent chez ces futurs enseignants (section 5).

\section{Notion de rapport à}

Après avoir brièvement défini ce que nous entendons par rapport à (point 2.1), nous nous questionnerons sur la pertinence d'importer ce concept pour les objets « grammaire » et «didactique de la grammaire» (2.2). Nous proposerons une définition du rapport à la grammaire et à la didactique de la grammaire (point 2.3) et expliquerons pourquoi nous avons choisi de nous intéresser au rapport à dans le cadre de notre recherche (point 2.4).

\subsection{Définition du rapport à}

Le Dictionnaire des concepts fondamentaux des didactiques (Reuter, Cohen-Azria, Daunay, Delcambre, \& Lahanier-Reuter, 2007) présente le concept de rapport à comme « la relation (cognitive, mais aussi sociopsychoaffective) qu'entretient l'apprenant avec les contenus et qui conditionne en partie l'apprentissage de ces derniers ${ }^{5}$ : un rapport aux contenus qui ne correspond pas à celui que l'école envisage peut rendre difficile l'accès aux contenus enseignés. » Comme Reuter et ses collaborateurs ne définissent pas ce qu'ils entendent par relation cognitive et sociopsychoaffective, nous nous tournons vers un texte fondateur pour éclairer cet aspect. Charlot (1997) propose le rapport épistémique au savoir, ce 
que nous interprétons comme le rapport à la connaissance et à son apprentissage. Pour ce qui est de la relation sociopsychoaffective, elle semble embrasser les deux autres rapports qui composent le rapport au savoir élaboré par Charlot (1997) : le rapport identitaire et social au savoir. Nous retenons également la définition qu'Émery-Bruneau (2010) donne du rapport identitaire, soit « le rapport du sujet à lui-même et à sa propre subjectivité. » Le rapport social au savoir apparait, quant à lui, comme le rapport du sujet aux autres, voire au monde. Dans cette optique, le rapport de l'apprenant au savoir n'est donc pas seulement lié à sa posture à l'égard des connaissances (rapport cognitif), mais aussi à sa disposition psychologique, affective et sociale.

Pour Barré-De Miniac (2000), le rapport à concerne non seulement « la disposition (d'une) personne à l'égard d'un objet », mais également sa disposition " à l'égard de la mise en œuvre pratique de cet objet dans la vie personnelle, culturelle, sociale et professionnelle » (Barré-De Miniac, 2000, p. 13). Enfin, le rapport à est pluriel en ce sens qu'il n'existe pas UN rapport à puisqu'il se fonde sur la rencontre entre un objet et le sujet qui entretient le rapport avec celui-ci (Barré-De Miniac, 2008) : en d'autres mots, il y a autant de rapports au savoir que d'objets et de sujets.

Anctil (2010) s'appuie sur les définitions de Reuter et ses collaborateurs et de Barré-De Miniac pour présenter le rapport des enseignants à l'erreur lexicale. Après avoir dépeint les notions d'attitudes, de représentations et de conceptions, il constate que la notion de rapport à est plus efficace en didactique puisqu'elle «permet d'englober les différents aspects évoqués plus haut (c.f. les notions d'attitude, de représentation et de conception) en envisageant les contenus scolaires du point de vue de l'apprenant » (Anctil, 2010, p. 169). Chartrand et Prince (2009) adoptent la définition du rapport à élaborée par BarréDe Miniac et constatent que leur objet d'étude, l'écrit, n'est pas essentiellement de nature scolaire, mais aussi un objet de nature sociale. Il semble donc que le rapport à ne porte pas exclusivement sur des objets scolaires, mais également sur des objets sociaux.

En didactique du français, la notion de rapport à s'est déclinée, à l'origine, de différentes manières : le rapport à l'écriture, le rapport à l'écrit et le rapport à la culture. Voici une définition de ces notions, telle qu'elle apparait dans les travaux des chercheurs qui les ont introduites.

\begin{tabular}{cl}
\hline & \multicolumn{1}{c}{ Définitions $^{6}$} \\
\hline $\begin{array}{c}\text { Rapport } \\
\text { à l'écriture }\end{array}$ & $\begin{array}{l}\text { "L'ensemble des significations construites par le scripteur à propos de l'écriture, } \\
\text { de son apprentissage et de ses usages. » (Barré-De Miniac, 2002a, p. 29) }\end{array}$ \\
$\begin{array}{c}\text { Rapport } \\
\text { à l'écrit }\end{array}$ & $\begin{array}{l}\text { «Une relation de sens (...) entre un sujet (...) et l'écrit }{ }^{7} \text { dans toutes ses } \\
\text { dimensions. » (Chartrand \& Blaser, 2008, p. 111) }\end{array}$ \\
$\begin{array}{c}\text { Rapport } \\
\text { à la culture }\end{array}$ & $\begin{array}{l}\text { «Un ensemble de relations dynamiques d'un sujet situé avec des savoirs, des } \\
\text { objets, des acteurs et des pratiques culturels. » (Falardeau \& Simard, 2004, p. 5) }\end{array}$ \\
\hline
\end{tabular}

Dans la définition de Chartrand et Blaser (2008), il est question de « dimensions » : pour ces chercheuses, le rapport à l'écrit se décline en quatre dimensions, soit la dimension affective, la dimension axiologique, la dimension conceptuelle et la dimension praxéologique. Barré-De Miniac (2000, 2002a, 2008) a également opérationnalisé le rapport à l'écriture en quatre dimensions principales : l'investissement de l'écriture; les opinions et les attitudes; les conceptions de l'écriture et de son apprentissage ainsi que les modes d'investissement. Enfin, Falardeau et Simard (2004) ont, quant à eux, élaboré trois dimensions que tout rapport à la culture renferme : la dimension épistémique; la dimension subjective et la dimension sociale. Notons que Barré-De Miniac précise à maintes reprises que la liste de ces dimensions pourrait être modifiée et qu'il faut absolument concevoir les dimensions dans leurs interrelations et non les aborder de façon isolée (Reuter \& coll., 2007; Barré-De Miniac, 2008).

Plus récemment, le rapport à s'est décliné de nouvelles façons en didactique du français : il est désormais question de rapport à l'erreur lexicale (Anctil, 2010), de rapport à la lecture (Dionne, 2010) et de rapport à la lecture littéraire (Émery-Bruneau, 2010). Dans ce contexte, il nous apparait nécessaire de nous interroger sur la pertinence d'importer la notion de rapport à vers d'autres objets de savoir propres à 
la didactique du français, dont les objets « grammaire » et « didactique de la grammaire », objets au cœur de notre recherche.

\subsection{La notion de rapport à peut-elle être importée vers d'autres objets ?}

Quelques réserves sont émises par Reuter et ses collaborateurs (2007) ainsi que par Chartrand et Blaser (2008) quant au transfert des dimensions principales du rapport à l'écrit ou à l'écriture à d'autres contenus d'enseignement telles la lecture ou la communication orale. Les auteurs constatent que les transferts ne peuvent se faire de façon mécanique puisque les caractéristiques du rapport à l'écriture ou à l'écrit ne peuvent pas nécessairement s'appliquer au rapport à la lecture, par exemple (Reuter \& al., 2007).

Toutefois, certaines recherches récentes tendent à démontrer le contraire. En effet, Dionne (2010) applique directement la notion de rapport à l'écriture à la lecture : toutes les dimensions du rapport à l'écriture sont ainsi reprises telles quelles en interchangeant simplement le mot écriture par celui de lecture. D'autres recherches très étoffées comme celle d'Émery-Bruneau (2011) sur le rapport à la lecture littéraire puisent dans plusieurs notions pour élaborer leur cadre théorique. Émery-Bruneau (2011) s'inspire du rapport à la culture (Falardeau \& Simard, 2004), du rapport à l'écrit (Chartrand \& Blaser, 2008) et du rapport au savoir développé par l'Équipe ESCOL (Charlot, 1997) pour construire et articuler la notion de rapport à la lecture littéraire. En effet, le rapport à la lecture littéraire reprend entre autres les dimensions subjective, épistémique et sociale du rapport à la culture et intègre la dimension praxéologique puisée dans le modèle du rapport à l'écrit. Finalement, Anctil (2010) croit qu'en circonscrivant adéquatement l'objet du rapport à étudié (dans son étude, le rapport à l'erreur lexicale), les dimensions retenues pour sa caractérisation seront pertinentes. Il cite en exemple la dimension affective qu'il a choisi d'évacuer considérant qu'elle n'est pas appropriée pour caractériser le rapport à l'erreur lexicale alors qu'elle aurait été adéquate dans une étude sur le rapport à l'erreur en général.

À nos yeux, la notion de rapport à peut être importée uniquement s'il s'agit d'un rapport à un objet de savoir, scolaire ou social. Nous justifions notre position en fonction de l'origine même de la notion, soit celle de rapport au savoir (Charlot, 1997). En ce sens, nous prenons certaines distances à l'égard de la notion de rapport à l'erreur lexicale (Anctil, 2010) puisque, selon nous, l'erreur lexicale ne constitue pas, à proprement parler, un objet de savoir.

Si nous adhérons à l'idée que la notion de rapport à puisse être importée vers de nouveaux objets de savoir, nous sommes plus prudents quant à l'importation des dimensions (affective, subjective, etc.). En effet, selon nous, ces dimensions dépendent à la fois de l'objet de savoir étudié et des objectifs de la recherche : elles sont des notions en constante évolution et des outils euristiques qui ont permis d'analyser des données de recherche en didactique et ainsi d'identifier des variables pertinentes pour la recherche et le travail didactique (Chartrand \& Blaser, 2008 ; Barré-De Miniac, 2008). Pour ces raisons, nous retenons, pour l'instant, une définition générale du rapport à la gramamire et du rapport à la didactique de la grammaire.

\subsection{Définition du rapport à la grammaire et du rapport à la didactique de la grammaire}

Nous nous inspirons largement de la définition proposée de Barré-De Miniac $(2002 b)^{8}$ pour définir les notions de rapport à la grammaire et de rapport à la didactique de la grammaire. Pour répondre à notre objectif de recherche, soit décrire les rapports à la grammaire et les rapports à la didactique de la grammaire qu'entretiennent de futurs enseignants de français, nous définissons cette notion ainsi :

La disposition d'un futur enseignant à l'égard des objets «grammaire » et « didactique de la grammaire », de même qu'à l'égard de la mise en œuvre pratique de ces objets dans sa vie personnelle (d'apprenant) et sa vie professionnelle (d'enseignant). 
Ainsi, les futurs enseignants ont un double statut à l'égard de ces objets : celui d'apprenant, qui doit s'approprier des savoirs en grammaire et en didactique de la grammaire, et celui d'enseignant, qui doit utiliser ces savoirs en situation d'enseignement, notamment lors des stages de formation.

\subsection{Pourquoi s'intéresser au rapport à la grammaire et à sa didactique ?}

Reuter et collaborateurs (2007) et Barré-De Miniac (2002b) insistent fortement sur l'importance d'intervenir sur le rapport à dans l'optique de reconstruire des rapports au savoir qui soient plus adéquats pour les apprenants, surtout pour ceux qui auraient développé des rapports aux contenus qui ne favoriseraient pas l'apprentissage. Dans ce sens, intervenir sur les rapports à la grammaire et les rapports à la didactique de la grammaire des étudiants au programme de formation à l'enseignement du français s'avère tout indiqué. Et comme les résultats de la recherche menée par Falardeau et Simard (2009) montrent que la majorité des enseignants interrogés ont avoué avoir développé une relation négative à l'égard de la grammaire et de son enseignement dans le cadre de leurs études secondaires (Falardeau \& Simard, 2009) , nous sommes convaincus de la pertinence d'étudier le rapport à la grammaire et à la didactique de la grammaire.

Alors que Reuter et collaborateurs (2007) l'abordent davantage sous l'angle des apprenants, Barré-De Miniac (2000) réserve un chapitre entier de son ouvrage intitulé Le rapport à l'écriture: aspects théoriques et didactiques à promouvoir l'importance du questionnement du rapport à chez les futurs enseignants afin qu'ils soient plus au fait de leur propre rapport à l'écriture. Elle affirme que de ne pas prendre en compte cet élément pourrait amener certains enseignants à projeter inconsciemment leur propre rapport à sur leurs élèves. Ainsi, l'enseignant qui agirait de la sorte n'aurait pas accès au rapport $\grave{a}$ qui diffère du sien et rencontrerait certaines difficultés à bien accompagner ses élèves dans leurs apprentissages. Dans un contexte universitaire de formation à l'enseignement du français, il est essentiel, pour les futurs enseignants, de connaitre ou de comprendre leur propre rapport à la grammaire et à la didactique de la grammaire afin de les comprendre et de les modifier, le cas échéant.

\section{Méthodologie}

Afin de décrire les rapports à la grammaire et les rapports à la didactique de la grammaire de futurs enseignants, nous avons conçu un questionnaire que nous avons administré à 49 étudiants ${ }^{10}$ d'une université québécoise à la fin du cours Didactique de la grammaire. Le cours de didactique de la grammaire comprend 45 heures de cours et compte pour 3 des 120 crédits universitaires du programme en enseignement au secondaire. Ce cours est offert après 2 cours de grammaire, qui totalisent 90 heures de cours et 6 crédits universitaires. Ainsi, au moment de remplir le questionnaire, les étudiants avaient terminé leur formation universitaire en grammaire et en didactique de la grammaire.

Le questionnaire comprend 57 questions, dont 36 fermées et 21 ouvertes. Le questionnaire est rempli en classe en environ 75 minutes, via l'outil en ligne SurveyMonkey: cet outil présente de nombreux avantages, notamment celui de pouvoir présenter aléatoirement les choix de réponses. Le questionnaire comporte quatre sections. La troisième section vise à établir un portrait des savoirs en grammaire et en didactique de la grammaire (20 questions $)^{11}$, la quatrième à obtenir des données sociodémographiques pour chacun des étudiants, entre autres l'âge, le sexe, la langue première et l'expérience en enseignement (10 questions). Les deux premières sections portent sur le rapport à la grammaire et sur le rapport à la didactique de la grammaire et sont celles qui nous intéressent dans le cadre de cet article.

Les sections 1 et 2 contiennent un total de 27 questions, 10 sur le rapport à la grammaire, 16 sur le rapport à la didactique de la grammaire et 1 sur les deux objets; elles distinguent l'étudiant selon deux rôles, en tant qu'apprenant (section 1) et en tant que futur enseignant (section 2). L'analyse des réponses aux questions de la troisième section du questionnaire est effectuée en fonction du type de question posée (fermée ou ouverte). Les questions ouvertes (7/27) sont analysées de façon qualitative à l'aide d'un système de codage ouvert; les questions fermées (20/27) donnent lieu à des analyses descriptives (fréquence, moyenne) et inférencielles non paramétriques (test des rangs signés de Wilcoxon, corrélation 
de Spearman, Khi-Carré) Nous présenterons les résultats préliminaires récoltés à 15 questions de ces deux sections. Notons que la recherche est exploratoire et descriptive en ce qu'elle tente d'établir un portrait des rapports à deux objets, la grammaire et la didactique de la grammaire, qui n'ont pas fait l'objet de recherches antérieures. Ainsi, les résultats ne peuvent prétendre à une quelconque généralisation : des travaux futurs sur un échantillon plus large nous permettront de proposer des descriptions et des interprétations plus précises, voire nuancées.

\section{Résultats}

\subsection{La grammaire, c'est...}

Nous avons d'abord tenté de faire émerger les rapports à la grammaire des étudiants en enseignement du français au secondaire grâce à une question ouverte: Notez spontanément trois mots ou courtes expressions qui complètent le mieux l'énoncé suivant et ordonnez-les selon l'importance que vous leur accordez. Pour moi, en tant qu'étudiant, et non en tant qu'enseignant, la grammaire, c'est... (question $4)^{12}$. Le tableau 1 présente les réponses au premier des trois termes mentionnés par les étudiants.

Tableau 1

Pour des étudiants, la grammaire, c'est... ( $1^{\mathrm{er}}$ terme $)$

\begin{tabular}{|c|c|c|c|c|}
\hline Réponses axées sur. & \multicolumn{3}{|c|}{ Fréquences } & Total \\
\hline \multirow[t]{4}{*}{ L'objet de savoir } & \multicolumn{2}{|c|}{ Un objet spécifique } & 9 & \multirow{4}{*}{18} \\
\hline & \multicolumn{2}{|c|}{ Un système } & 5 & \\
\hline & \multicolumn{2}{|l|}{ Des règles } & 2 & \\
\hline & \multicolumn{2}{|l|}{ Autres } & 2 & \\
\hline \multirow[t]{2}{*}{ Les valeurs ou les sentiments } & \multirow{2}{*}{\multicolumn{2}{|c|}{$\begin{array}{l}\text { Positits } \\
\text { Négatifs }\end{array}$}} & 11 & \multirow{2}{*}{17} \\
\hline & & & 6 & \\
\hline \multirow[t]{4}{*}{ La fonction } & \multirow[t]{3}{*}{ Sert d'outil... } & d'analyse & 3 & \multirow{4}{*}{11} \\
\hline & & en général & 3 & \\
\hline & & d'apprentissage & 2 & \\
\hline & \multicolumn{2}{|c|}{ Sert en écriture } & 3 & \\
\hline \multicolumn{4}{|l|}{ Non-Analysable } & 3 \\
\hline & & & Total & 49 \\
\hline
\end{tabular}

Les étudiants pensent que la grammaire est un objet de savoir (18/49) qui consiste en un objet spécifique $(9 / 18)$, un système $(5 / 18)$ ou des règles $(2 / 18)$. Un nombre presque égal d'étudiants $(17 / 49)$ ne répondent pas en fonction de la définition de l'objet, mais plutôt en fonction de leur perception de l'objet, en précisant la valeur ou le sentiment qu'ils y attribuent; ce résultat est d'autant plus intéressant que la question ne favorisait pas, à priori, l'émergence de cette dimension (nous reviendrons sur les dimensions à la section 5). Les valeurs et les sentiments attribués à la grammaire sont tantôt positifs $(11 / 17)$ tantôt négatifs (6/17) : les termes important (3/17), utile (2/17), essentielle (1/17), nécessaire (1/17), amusant $(1 / 17)$, une matière fondamentale $(1 / 17)$, une notion importante $(1 / 17)$, essentiel pour toutes les matières $(1 / 17)$ traduisent une valeur positive ; les termes complexe $(2 / 17)$, difficile $(2 / 17)$, un peu ennuyant $(1 / 17)$ et ce n'est pas assez bien appris (1/17), une valeur négative. Enfin, pour certains, la grammaire a une fonction (11/49), soit celle d'être un outil (8/11), plus précisément un outil d'analyse (3/11), un outil au sens général (3/11), un outil d'apprentissage (2/11); pour d'autres, la grammaire est un outil au service de l'écriture (3/11). Aux $2^{\mathrm{e}}$ et $3^{\mathrm{e}}$ termes, les réponses axées sur les valeurs surclassent les réponses orientées vers l'objet de savoir, comme le montre le tableau 2.

\section{Tableau 2}

Pour des étudiants, la grammaire, c'est... ( $2^{\mathrm{e}}$ et $3^{\mathrm{e}}$ termes $)$ 


\begin{tabular}{|c|c|c|c|c|}
\hline \multirow[t]{2}{*}{ Réponses axées sur... } & & & \multicolumn{2}{|c|}{ Fréquences } \\
\hline & & & $2^{\mathrm{e}}$ terme & $3^{\mathrm{e}}$ terme \\
\hline \multirow{3}{*}{$\begin{array}{l}\text { Les valeurs et } \\
\text { sentiments }\end{array}$} & \multicolumn{2}{|l|}{ Positifs } & 6 & 10 \\
\hline & \multicolumn{2}{|l|}{ Neutres } & 1 & 2 \\
\hline & \multicolumn{2}{|c|}{ Négatifs } & 14 & 10 \\
\hline \multirow[t]{4}{*}{ L'objet de savoir } & \multicolumn{2}{|c|}{ Un objet spécifique } & 10 & 9 \\
\hline & \multicolumn{2}{|c|}{ Un système } & 4 & 1 \\
\hline & \multicolumn{2}{|l|}{ Des règles } & 3 & 1 \\
\hline & \multicolumn{2}{|l|}{ En général } & 2 & 4 \\
\hline \multirow[t]{7}{*}{ La fonction } & \multirow[t]{4}{*}{ Sert d'outil... } & d'analyse & 3 & 1 \\
\hline & & en général & 1 & 2 \\
\hline & & d'apprentissage & 0 & 0 \\
\hline & & pour comprendre & 1 & 0 \\
\hline & \multicolumn{2}{|c|}{ Sert en écriture } & 3 & 3 \\
\hline & \multicolumn{2}{|c|}{ Sert à enseigner } & 0 & 2 \\
\hline & \multicolumn{2}{|c|}{ Sert à l'écriture et à la lecture } & 0 & 1 \\
\hline \multicolumn{3}{|l|}{ La norme } & 1 & 1 \\
\hline
\end{tabular}

$\mathrm{Au} 2^{\mathrm{e}}$ terme, une catégorie disparaît, la grammaire un outil pour apprendre, au profit de deux nouvelles catégories, la grammaire est un outil pour comprendre (1 occurrence) et la grammaire est une norme (1 occurrence). $\mathrm{Au} 3^{\mathrm{e}}$ terme, deux nouvelles catégories émergent: la grammaire sert à enseigner $(2$ occurrences) et à l'écriture et à la lecture (1 occurrence). Si les réponses axées sur les valeurs et les sentiments sont très présentes aux trois termes, elles sont dominantes aux deux derniers (respectivement 21 et 22 occurrences sur sur 49). Nous retiendrons donc de ces analyses (tableaux 1 et 2) que la grammaire est un objet d'apprentissage auquel les étudiants attribuent spontanément une valeur ou un sentiment généralement positif au $1^{\mathrm{er}}$ terme, mais qui se transforme vers une valeur négative aux $2^{\mathrm{e}}$ et $3^{\mathrm{e}}$ termes.

Une question visait précisément à cerner la perception des étudiants envers leur apprentissage de la grammaire : Globalement, pendant votre formation universitaire, comment avez-vous perçu votre propre apprentissage de la grammaire? Cochez votre choix pour chacun des adjectifs (question 7). Parmi les 13 adjectifs, 7 ont une connotation positive (satisfaisant, passionnant, valorisant, amusant, stimulant, agréable, utile), 6 une connotation négative (frustrant, difficile, ennuyant, pénible, contraignant, angoissant). Pour chacun de ces adjectifs, les étudiants devaient choisir leur degré d'accord sur une échelle de Likert de 1 à 4 (pas du tout d'accord, un peu d'accord, plutôt d'accord, tout à fait d'accord). Le graphique 1 présente le degré d'accord des répondants pour chacun des adjectifs.

Graphique 1

Perceptions d'étudiants à l'égard de leur apprentissage de la grammaire 


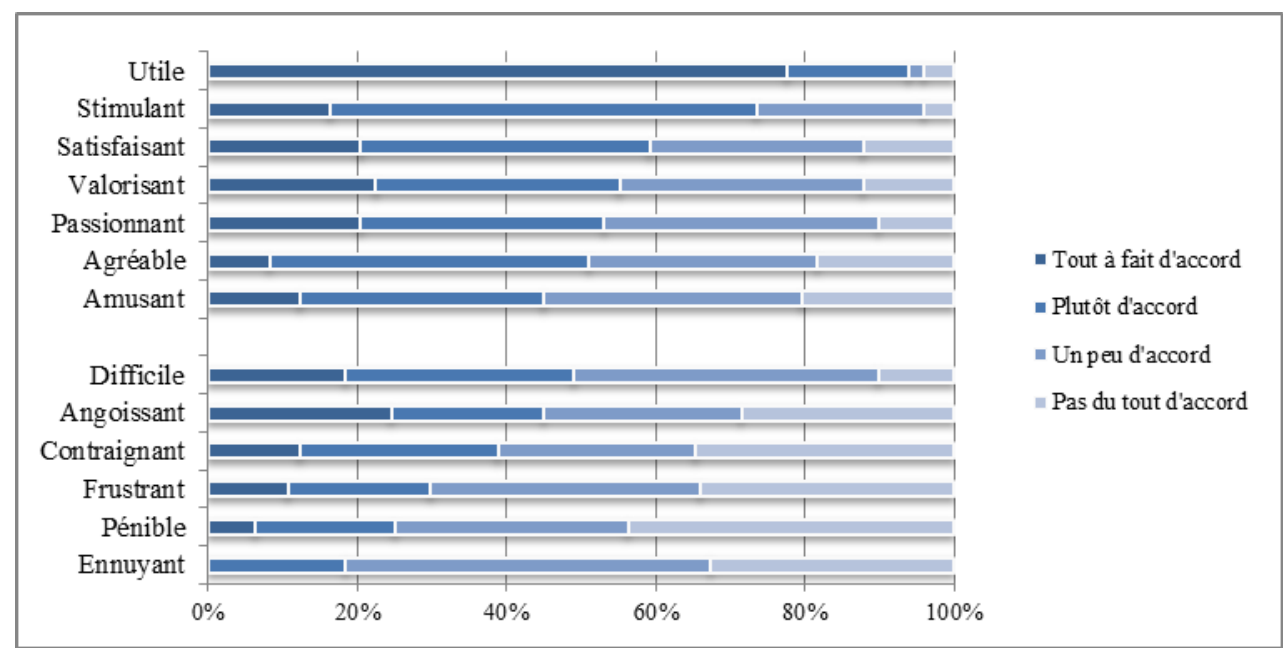

Plus de 3 étudiants sur 10 ont trouvé leur apprentissage de la grammaire pas du tout pénible $(43,8 \%)$, contraignant $(34,7 \%)$, frustrant (34\%) et ennuyant (32,7\%). À l'opposé, plus de 4 étudiants sur 10 ont trouvé leur apprentissage plutôt ou tout à fait difficile (49\%) et angoissant (44,9\%). Bonne nouvelle : plus de 7 étudiants sur 10 sont tout à fait d'accord avec le fait que leur apprentissage de la grammaire est utile $(77,6 \%)$ et stimulant $(73,5 \%)$ et aucun n'estime que son apprentissage de la grammaire a été tout à fait ennuyant. Amusant semble être l'adjectif qui décrit le moins bien leur apprentissage de la grammaire (20,41\% pas du tout d'accord). Enfin, l'adhésion (degré d'accord à notre échelle de Likert de 1 à 4$)$ aux adjectifs positifs est significativement plus élevée que l'adhésion aux adjectifs négatifs (moyennes $=$ $2,75 / 4$ vs $2,16 / 4$; test de rangs signés de Wilcoxon, $\mathrm{p}=0,002)$ ).

Nous nous sommes également intéressés à leur perception de l'enseignement de la grammaire. Ainsi, nous avons posé une question équivalente à la question précédente, mais en insistant pour que les étudiants adoptent une posture de futur enseigant : En tant que futur enseignant, comment envisagez-vous votre futur enseignement de la grammaire? Cochez votre choix pour chacun des adjectifs (question 17). Le graphique 2 présente le degré d'accord des répondants pour chacun des adjectifs.

Graphique 2

Perceptions de futurs enseignants à l'égard de leur futur enseignement de la grammaire ${ }^{13}$

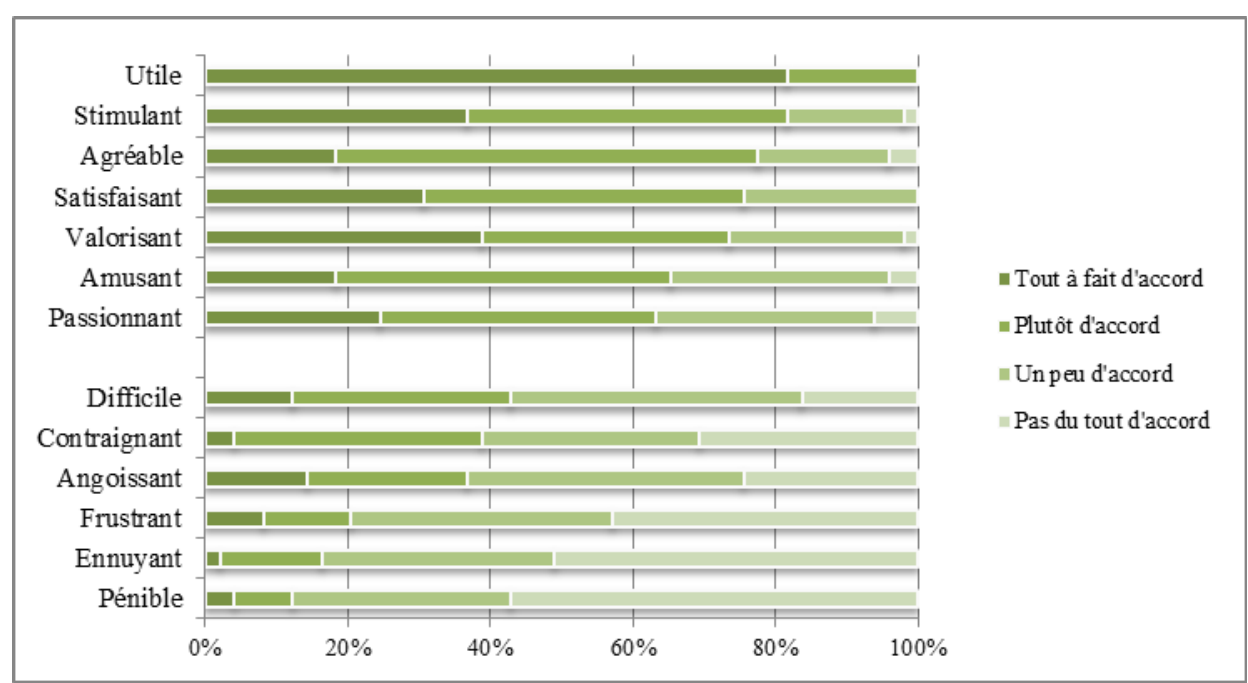


Tous les étudiants $(100 \%)$ estiment que leur enseignement de la grammaire sera plutôt ou tout à fait utile. Bonne nouvelle pour les générations d'élèves à venir : plus de 8 étudiants sur 10 soutiennent que leur enseignement de la grammaire sera plutôt ou tout à fait stimulant $(81,6 \%), 7$ sur 10 agréable $(77,48 \%)$, satisfaisant $(75,5 \%)$ et valorisant $(73,5 \%)$. Un peu plus de 5 étudiants sur 10 n'envisagent pas du tout que leur enseignement soit ennuyant (51\%), alors que seulement 3 sur 10 estiment que leur apprentissage de la grammaire n'a pas du tout été ennuyant $(32,7 \% \text {, graphique } 1)^{14}$. De plus, certains prévoient que l'enseignement de la grammaire sera tout à fait ou plutôt difficile $(42,9 \%)$, contraignant $(38,8 \%)$ et angoissant $(36,7 \%)$, et ce, même s'ils l'envisagent amusant $(65,3 \%)$ et passionnant $(63,3 \%)$. Près de 9 étudiants sur 10 ne sont cependant pas du tout ou peu d'accord avec le fait que l'enseignement de la grammaire sera pénible (87,8\%). Enfin, l'adhésion aux adjectifs positifs est significativement plus élevée que l'adhésion aux adjectifs négatifs (moyennes $=3,1 / 4$ vs $2 / 4$; test de rangs signés de Wilcoxon, $\mathrm{p}<$ 0,0001).

Les corrélations de Spearman montrent que les degrés d'accord entre la posture étudiante et enseignante ne sont pas significativement corrélés pour la majorité des adjectifs seulement (7/13). Pour les adjectifs angoissant $(\mathrm{Rho}=0,27, \mathrm{p}=0,07)$, difficile $(\mathrm{Rho}=0,25, \mathrm{p}=0,09)$, contraignant $(\mathrm{Rho}=0,25, \mathrm{p}=0,09)$, valorisant $(R h o=0,2, p=0,17)$, satisfaisant $(R h o=0,16, p=0,26)$, frustrant $(R h o=0,14, p=0,35)$ et utile (Rho $=-0,02, p=0,9$ ), aucune différence significative n'a été observée selon la posture des répondants. Par contre, plus un répondant est en accord avec les adjectifs stimulant $(\mathrm{Rho}=0,3, \mathrm{p}=0,04)$, ennuyant $(\mathrm{Rho}=0,31, \mathrm{p}=0,03)$, agréable $(\mathrm{Rho}=0,33, \mathrm{p}=0,02)$, passionnant $(\mathrm{Rho}=0,35, \mathrm{p}=0,01)$, amusant (Rho $=0,46, \mathrm{p}=0,001)$ et pénible ( $\mathrm{Rho}=0,55 \mathrm{p}<0,001)$ quand il répond dans la posture d'étudiant, plus il est en accord avec ces mêmes adjectifs dans la posture d'enseignant. Bien que significatifs, ces coefficients de corrélation sont relativement faibles (éloignés de 1). Outre ces corrélations, il est intéressant de constater que l'adhésion aux adjectifs positifs comme futur enseignant est significativement plus élevée que celle comme étudiant (test de rangs signés de Wilcoxon, $p=0,001$ ). Ces résultats suggèrent que les étudiants perçoivent la grammaire plus posivement comme enseignant que comme apprenant ce que montre le graphique 3.

\section{Graphique 3}

Comparaison de la perception d'étudiants à l'égard de leur apprentissage et de leur futur enseignement de la grammaire selon la valence des adjectifs

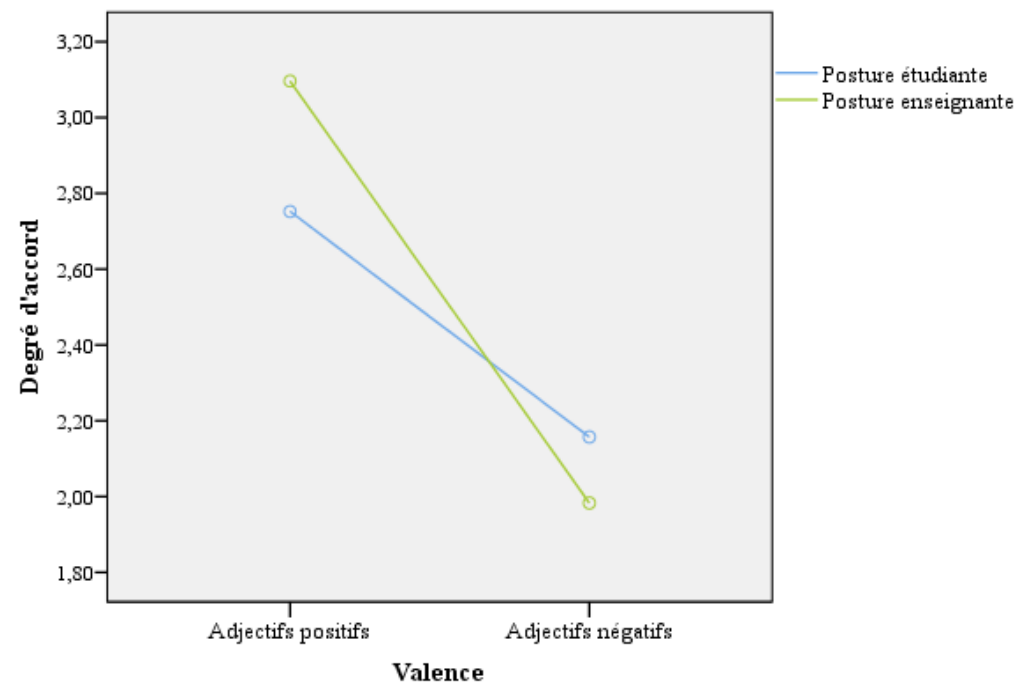

\subsection{La grammaire sert à...}


Une question ouverte nous a permis de faire émerger les finalités que les étudiants attribuent à la grammaire. Le tableau 3 présente les réponses au premier des trois termes mentionnés par les étudiants à la question Notez spontanément trois mots ou courtes expressions qui complètent le mieux l'énoncé suivant et ordonnez-les selon l'importance que vous leur accordez. Pour moi, en tant qu'étudiant, et non en tant qu'enseignant, la grammaire, sert à... (question 5).

Tableau 3

Pour les étudiants, la grammaire, sert à... $\left(1^{\mathrm{re}}\right.$ terme $)$

\begin{tabular}{|c|c|c|c|c|c|}
\hline \multicolumn{3}{|l|}{ Réponses axées sur.. } & \multicolumn{2}{|c|}{ Fréquences } & \multirow[t]{2}{*}{ Total } \\
\hline \multirow[t]{10}{*}{ La maitrise } & \multirow[t]{4}{*}{ de l'écriture } & Bien écrire & 6 & \multirow{4}{*}{18} & \\
\hline & & Mieux écrire & 5 & & \multirow{9}{*}{34} \\
\hline & & Écrire sans fautes & 5 & & \\
\hline & & En général & 2 & & \\
\hline & de la langue & En général & 5 & \multirow{2}{*}{8} & \\
\hline & & En se corrigeant & 3 & & \\
\hline & \multicolumn{2}{|c|}{ de la communication } & & 3 & \\
\hline & \multicolumn{2}{|c|}{ à l'oral } & & 2 & \\
\hline & \multicolumn{2}{|l|}{ des règles } & & 2 & \\
\hline & \multicolumn{2}{|c|}{ d'un objet spécifique } & & 1 & \\
\hline \multirow[t]{3}{*}{ La compréhension } & \multicolumn{2}{|c|}{ du fonctionnement de la langue } & & 7 & \multirow{3}{*}{11} \\
\hline & \multicolumn{2}{|c|}{ en général } & & 3 & \\
\hline & \multicolumn{2}{|l|}{ de la langue } & & 1 & \\
\hline \multicolumn{3}{|l|}{ L'analyse } & & & 2 \\
\hline \multicolumn{3}{|c|}{ La performance scolaire } & & & 1 \\
\hline \multirow{2}{*}{\multicolumn{3}{|c|}{ Un outil }} & & & 1 \\
\hline & & & & Total & 49 \\
\hline
\end{tabular}

Les résultants suggèrent que, pour les étudiants, la grammaire permet de mieux maitriser la langue (34/49), de comprendre (11/49), d'analyser la langue (2/49), de performer académiquement (1/49) ou de l'utiliser comme un outil de référence (1/49). Leur vision de la maitrise de la langue est liée à la maitrise de l'écriture (18/34), de la langue en général (8/34), de la communication en générale (3/19 3/34), de l'oral (2/34), des règles (2/34) et d'un objet spécifique (1/34). La compréhension que permet, selon eux, la grammaire touche au fonctionnement de la langue (7/49), à la compréhension en général (3/49) et à la compréhension de la langue (1/49). Aux $2^{\mathrm{e}}$ et $3^{\mathrm{e}}$ termes, les tendances dans les réponses des étudiants se confirment, comme le montre le tableau 2.

Tableau 4

Pour les étudiants, la grammaire, sert à... ( $2^{\mathrm{e}}$ et $3^{\mathrm{e}}$ termes $)$

\begin{tabular}{|llcc|}
\hline Réponses axées sur... & & \multicolumn{2}{c|}{ Fréquences } \\
\hline \multirow{2}{*}{ La maitrise } & & $2^{\mathrm{e}}$ terme & $3^{\mathrm{e}}$ terme \\
\cline { 2 - 4 } & de l'écriture & 12 & 9 \\
\cline { 2 - 4 } & de la langue en général & 3 & 12 \\
\cline { 2 - 4 } & de la communication en général & 3 & 6 \\
\cline { 2 - 4 } & de l'oral & 3 & 2 \\
\hline La compréhension & d'un la lecture & 1 & 0 \\
\hline & de la langue & 2 & 0 \\
\hline
\end{tabular}




\begin{tabular}{|llll|}
\hline & du fonctionnement de la langue & 3 & 3 \\
\cline { 2 - 4 } & en général & 3 & 1 \\
\cline { 2 - 4 } & d'un objet spécifique & 1 & 0 \\
\hline Expliquer la langue et son fonctionnement & 3 & 4 \\
\hline Prescription de l'usage & 4 & 1 \\
\hline La performance scolaire & 2 & 1 \\
\hline Non analysable & 2 & 1 \\
\hline Réponses variées (penser, apprendre, etc.) & 0 & 7 \\
\hline
\end{tabular}

$\mathrm{Au} 2^{\mathrm{e}}$ terme, l'idée selon laquelle la grammaire sert à maitriser la lecture apparait. Notons également que la grammaire servant à expliquer la langue et son fonctionnement ainsi qu'à prescrire l'usage font une timide entrée au $2^{\mathrm{e}}$ et $3^{\mathrm{e}}$ terme, alors que la grammaire pour analyser disparait. Bref, l'ensemble de ces résultats suggère que les étudiants estiment que la grammaire sert d'abord et avant tout à maitriser la langue.

Nous avons demandé aux étudiants Globalement, en tant qu'étudiant, vous utilisez vos connaissances en grammaire pour... (question 9) ainsi que En tant que futur enseignant, en situation d'enseignement, vous ferez de la grammaire pour que vous élèves puissent... (question 20). Les étudiants devaient déterminer leur degré d'accord sur une échelle de Likert de 1 à 4 (pas du tout d'accord, un peu d'accord, plutôt d'accord, tout à fait d'accord), avec 14 activités susceptibles de mobiliser des connaissances grammaticales. Les deux questions nous permettent de comparer les activités que les étudiants prétendent réaliser avec leurs connaissances grammaticales à celles qu'ils prévoient faire réaliser dans leur futur enseignement. Le tableau 5 présente les résultats à ces questions, selon la posture d'étudiant (question 9) et d'enseignant (question 20).

Tableau 5

Comparaison des activités soi-disant réalisées par des étudiants avec leurs connaissances grammaticales à celles prévues dans leur futur enseignement

\begin{tabular}{|c|c|c|c|c|c|c|c|c|}
\hline \multirow{2}{*}{$\begin{array}{l}\text { activités } \\
\text { Corriger la syntaxe }\end{array}$} & \multicolumn{2}{|c|}{$\begin{array}{c}\text { pas } \\
\text { d'accord }\end{array}$} & \multicolumn{2}{|c|}{$\begin{array}{l}\text { un peu } \\
\text { d'accord }\end{array}$} & \multicolumn{2}{|c|}{$\begin{array}{c}\text { plutôt } \\
\text { d'accord }\end{array}$} & \multicolumn{2}{|c|}{$\begin{array}{l}\text { tout à fait } \\
\text { d'accord }\end{array}$} \\
\hline & $0,0 \%$ & $2,0 \%$ & $2,0 \%$ & $4,1 \%$ & $14,3 \%$ & $26,5 \%$ & $83,7 \%$ & $67,4 \%$ \\
\hline Comprendre le fonctionnement de la langue & $0,0 \%$ & $2,0 \%$ & $6,1 \%$ & $4,1 \%$ & $10,2 \%$ & $12,2 \%$ & $83,7 \%$ & $81,6 \%$ \\
\hline Maitriser le fonctionnement de la langue & $0,0 \%$ & $0,0 \%$ & $4,1 \%$ & $6,1 \%$ & $18,4 \%$ & $18,4 \%$ & $77,6 \%$ & $75,5 \%$ \\
\hline Corriger l'orthographe grammaticale & $2,0 \%$ & $2,0 \%$ & $8,2 \%$ & $2,0 \%$ & $12,2 \%$ & $24,5 \%$ & $77,6 \%$ & $71,4 \%$ \\
\hline Bien écrire & $4,1 \%$ & $2,0 \%$ & $0,0 \%$ & $0,0 \%$ & $20,4 \%$ & $12,2 \%$ & $75,5 \%$ & $85,7 \%$ \\
\hline Comprendre des phrases & $8,2 \%$ & $0,0 \%$ & $12,2 \%$ & $2,0 \%$ & $12,2 \%$ & $22,5 \%$ & $67,3 \%$ & $75,5 \%$ \\
\hline Bien vous exprimer ${ }^{15}$ & $8,2 \%$ & $0,0 \%$ & $4,1 \%$ & $10,2 \%$ & $24,5 \%$ & $28,6 \%$ & $63,3 \%$ & $61,2 \%$ \\
\hline Corriger la ponctuation & $2,0 \%$ & $0,0 \%$ & $14,3 \%$ & $8,2 \%$ & $20,4 \%$ & $28,6 \%$ & $63,3 \%$ & $63,3 \%$ \\
\hline Produire des phrases & $4,1 \%$ & $2,0 \%$ & $8,2 \%$ & $6,1 \%$ & $26,5 \%$ & $20,4 \%$ & $61,2 \%$ & $71,4 \%$ \\
\hline Réussir un examen & $8,2 \%$ & $10,2 \%$ & $10,2 \%$ & $12,2 \%$ & $28,6 \%$ & $28,6 \%$ & $53,1 \%$ & $49.0 \%$ \\
\hline Être compris & $14,3 \%$ & $2,1 \%$ & $20,4 \%$ & $12,5 \%$ & $12,2 \%$ & $27,1 \%$ & $53,1 \%$ & $58,3 \%$ \\
\hline Vous aider à communiquer & $20,4 \%$ & $0,0 \%$ & $10,2 \%$ & $10,2 \%$ & $28,6 \%$ & $24,5 \%$ & $40,8 \%$ & $65,3 \%$ \\
\hline Corriger le lexique & $14,3 \%$ & $2,0 \%$ & $26,5 \%$ & $24,5 \%$ & $22,4 \%$ & $38,8 \%$ & $36,7 \%$ & $34,7 \%$ \\
\hline Vous aider à lire & $24,5 \%$ & $2,0 \%$ & $32,7 \%$ & $14,3 \%$ & $14,3 \%$ & $28,6 \%$ & $28,6 \%$ & $55,1 \%$ \\
\hline
\end{tabular}

Légende $:$ colonnes bleues $=$ posture étudiante, colonnes vertes $=$ posture enseignante

Nous constatons que tous les étudiants en enseignement du français disent utiliser leurs connaissances grammaticales pour comprendre le fonctionnement de la langue (tout à fait d'accord 83,7\%; plutôt d'accord 10,2\%, un peu d'accord 6,1\%), corriger la syntaxe (tout à fait d'accord $83,7 \%$; plutôt d'accord 
$14,3 \%$; un peu d'accord 2,0\%) et maitriser le fonctionnement de la langue (tout à fait d'accord 77,6\%; plutôt d'accord 18,4\% ; un peu d'accord 4,1\%); en situation d'enseignement, ce sont plutôt les activités maitriser le fonctionnement de la langue, comprendre les phrases, bien s'exprimer, corriger la ponctuation ou mieux communiquer qui sont prévues. Par ailleurs, plus de 8 étudiants sur 10 sont tout à fait ou plutôt d'accord pour dire qu'ils utilisent leurs connaissances grammaticales pour bien écrire (95,9\%), pour corriger l'orthographe grammaticale $(89,8 \%)$, pour bien s'exprimer $(87,8 \%)$, pour produire des phrases $(87,7 \%)$, pour corriger la pontuation $(83,7 \%)$ et pour réussir un examen $(81,7 \%)$; bien écrire $(97,9 \%)$, corriger la syntaxe $(93,9 \%)$, comprendre le fonctionnement de la langue $(93,8 \%)$ et produire des phrases $(91,8 \%)$ sont les activités visées par l'enseignement de la grammaire de plus de 9 futurs enseignants sur 10. Le graphique 4 compare la moyenne des adhésions aux quatre degrés d'accord de l'échelle de Likert, toujours selon les deux postures proposées aux questions 9 et 20.

Graphique 4

Comparaison des adhésions aux activités selon une posture d'étudiant ou d'enseignant

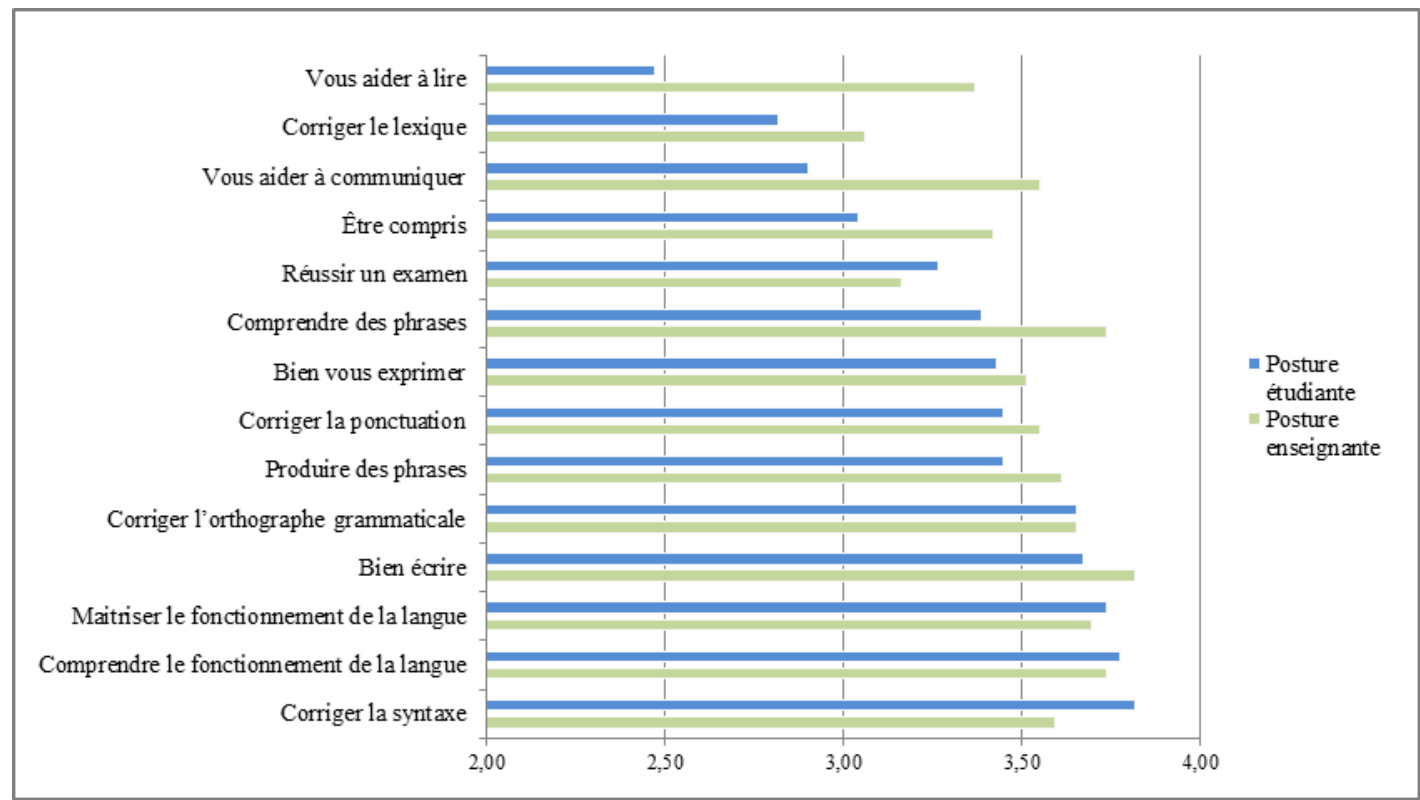

Des différences significatives s'observent pour 5 activités selon la posture que les répondants adoptent. En tant que futurs enseignants, ils estiment que les connaissances grammaticales des élèves permettront à ces derniers de mieux communiquer (moyenne: 3,55/4), de mieux lire (moyenne: 3,37/4), de comprendre des phrases (moyenne $=3,73 / 4$ ) et d'être compris (moyenne $=3,42 / 4$ ); pourtant, les étudiants sont moins d'accord pour utiliser leurs propres connaissances grammaticales pour réaliser ces mêmes activités (moyennes respectives : 2,90/4, 2,47/4, 3,39/4 et 3,04/4; test de rangs signés de Wilcoxon, p < 0,01). À l'opposé, les futurs enseignants de français sont davantage d'accord lorsqu'il s'agit d'utiliser la grammaire pour corriger leur propre syntaxe $(3,82 / 4)$ que lorsqu'il s'agit d'enseigner la grammaire pour que les élèves puissent corriger la syntaxe $(3,59 / 4)$, et ce, de façon significative (test de rangs signés de Wilcoxon, $\mathrm{p}=0,02$ ). Globablement, les étudiants ont choisi un plus fort degré d'accord pour l'ensemble des activités en tant qu'enseignant que pour les activités en tant qu'étudiant (moyenne = 3,53/4 vs 3,35/4; test de rangs signés de Wilcoxon, $\mathrm{p}=0,007$ ).

Le test du Khi-carré confirme que la distribution des réponses n'est pas aléatoire pour toutes les activités lorsqu'elles sont suggérées dans un contexte d'enseignement (Khi-2 > 15,898; $\mathrm{p}<0,001)$. Le même résultat est observé pour les activités en tant qu'étudiant (Khi-2 $>9,857, \mathrm{p}<0,05)$, à l'exception de corriger le lexique $($ Khi-2 $=5,122, \mathrm{p}=0,163)$ et aider à lire $(\mathrm{Khi}-2=3,65, \mathrm{p}=0,301)$ : ce résultat indique une plus grande variété dans les réponses des étudiants à ces deux activités, notamment en ce qui concerne l'utilisation de leurs connaissances en lecture. En fait, le lien entre la grammaire et les habiletés 
en lecture semble véritablement s'imposer qu'à 3 étudiants sur 49 (voir réponses répertoriées à la question 5, tableau 4). Une analyse par composantes principales sur un plus vaste échantillon nous permettra ultérieurement de déterminer dans quelles mesures les réponses des étudiants se regroupent autour de quelles catégories d'activités (par exemple, activités liées au fonctionnement de la langue vs celles liées à la correction ou à l'expression).

Parmi toutes les activités présentées au tableau 5, les étudiants devaient choisir, dans un premier temps, quelle activité ils estiment la plus importante en tant qu'étudiant (question 10) : près de 1 étudiant sur 2 estime que la maitrise du fonctionnement de la langue $(46,9 \%)$ est l'activité la plus importante, viennent ensuite en $2 \mathrm{e}$ et $3 \mathrm{e}$ positions comprendre le fonctionnement de la langue $(22,4 \%)$ et bien écrire $(8,2 \%)$. La question a été posée de nouveau, mais selon la posture enseignante (question 21) : les futurs enseignants de français disent faire de la grammaire surtout pour que leurs élèves puissent comprendre le fonctionnement de la langue $(30,6 \%)$, maitriser le fonctionnement de la langue $(26,5 \%)$ et bien écrire $(18,4 \%)$. Ainsi, pour seulement 1 répondant sur 5, comprendre le fonctionnement de la langue est l'activité la plus importante en tant qu'étudiant universitaire, alors que cette même activié, projetée dans un contexte d'enseignement, est jugée la plus importane pour 1 répondant sur 3. À l'inverse, la maitrise du fonctionnement de la langue est jugée l'activité la plus importante par près de la moitié des étudiants, alors que moins du tiers l'envisage comme l'activité la plus importante dans leur futur enseignement.

Les étudiants ont aussi été questionnés sur l'importance qu'ils accordaient à la grammaire, en insistant pour qu'ils prennent d'abord une posture étudiante (question 11), puis une posture enseignante (question 24). Ainsi, les questions suivantes leur ont été posées: Globalement, en tant qu'étudiant, vous trouvez important d'apprendre la grammaire pour... (question 11) et En tant que futur enseignant, quelle importance accorderez-vous aux aspects suivants dans l'apprentissage de la grammaire par vos élèves? (question 24). Les étudiants devaient choisir un niveau d'importance parmi 4 sur une échelle de Likert (pas important, peu important, plutôt important, très important), ou indiquer, pour la question 11 seulement, qu'aucun apprentissage n'avait été fait à ce sujet. Le tableau 6 présente les réponses à ces deux questions.

Tableau 6

Rôle de la grammaire selon les postures étudiante et enseignante

\begin{tabular}{|c|c|c|c|c|c|c|c|c|c|}
\hline \multirow{2}{*}{$\begin{array}{l}\text { aspects } \\
\text { La maîtrise du fonctionnement de la langue }\end{array}$} & \multirow{2}{*}{$\begin{array}{c}\begin{array}{c}\text { aucun } \\
\text { apprentissage }\end{array} \\
2,0 \%\end{array}$} & \multicolumn{2}{|c|}{$\begin{array}{c}\text { pas } \\
\text { important }\end{array}$} & \multicolumn{2}{|c|}{$\begin{array}{c}\text { peu } \\
\text { important }\end{array}$} & \multicolumn{2}{|c|}{$\begin{array}{c}\text { plutôt } \\
\text { important }\end{array}$} & \multicolumn{2}{|c|}{$\begin{array}{c}\text { très } \\
\text { important }\end{array}$} \\
\hline & & $0,0 \%$ & $0,0 \%$ & $4,1 \%$ & $4,1 \%$ & $16,3 \%$ & $30,6 \%$ & $77,6 \%$ & $65,3 \%$ \\
\hline La réutilisation de notions grammaticales en écriture & $0,0 \%$ & $0,0 \%$ & $0,0 \%$ & $0,0 \%$ & $0,0 \%$ & $24,5 \%$ & $8,2 \%$ & $75,5 \%$ & $91,8 \%$ \\
\hline La compréhension du fonctionnement de la langue & $0,0 \%$ & $0,0 \%$ & $0,0 \%$ & $8,2 \%$ & $2,0 \%$ & $16,3 \%$ & $20,4 \%$ & $75,5 \%$ & $77,6 \%$ \\
\hline L'utilisation du métalangage & $2,1 \%$ & $6,3 \%$ & $4,1 \%$ & $20,8 \%$ & $20,4 \%$ & $39,6 \%$ & $46,9 \%$ & $31,3 \%$ & $28,6 \%$ \\
\hline La réutilisation des notions grammaticales en lecture & $0,0 \%$ & $8,2 \%$ & $2,0 \%$ & $24,5 \%$ & $14,3 \%$ & $38,8 \%$ & $30,6 \%$ & $28,6 \%$ & $53,1 \%$ \\
\hline
\end{tabular}

Peu importe la posture, tous les étudiants estiment qu'il est très important ou plutôt important d'apprendre la grammaire pour réutiliser les notions grammaticales en écriture. Pour ce qui est de la posture d'étudiant, un peu plus de 9 répondants sur 10 croient qu'il leur est très important d'apprendre la grammaire pour maitriser le fonctionnement de la langue (très important 77,6\%; plutôt important 16,3\%) et comprendre le fonctionnement de la langue (très important 75,5\% ; plutôt important 16,3\%). Même si l'item portant sur la création de liens entre les notions grammaticales a été jugé très important par moins d'étudiants $(65,3 \%)$, l'adhésion y demeure élevée, ayant été jugé très ou plutôt important par 94\% des étudiants. Trois aspects revêtent une importance moindre pour eux; seulement 1 étudiant sur 3 pense que l'utilisation du métalangage (31\%) ainsi que la réutilisation de notions grammaticales à l'oral (35\%) et en lecture $(29 \%)$ sont très importants. En tant qu'enseignants, les répondants ont accordé de l'importance à 
la maitrise du fonctionnement de la langue (très important $65,3 \%$; plutôt important 30,6\%) et à la compréhension du fonctionnement de la langue (très important 77,6\% ; plutôt important 20,4\%) dans l'apprentissage de la grammaire de leurs élèves. La création de liens entre les notions grammaticales est très importante pour 3 étudiants sur 4 pour leur futur enseignement de la grammaire (très important $75,5 \%$ ), mais près du quart d'entre eux (pas important $4,1 \%$; peu important $20,4 \%$ ) attache moins d'importance à l'utilisation du métalangage en situation d'enseignement. Le graphique 5 compare les moyennes obtenues, sur une possibilité de 4 sur l'échelle de Likert, pour chacun des aspects et selon les deux postures.

Graphique 5

Comparaison des adhésions aux rôles à attribuer à la grammaire selon une posture d'étudiant ou d'enseignant

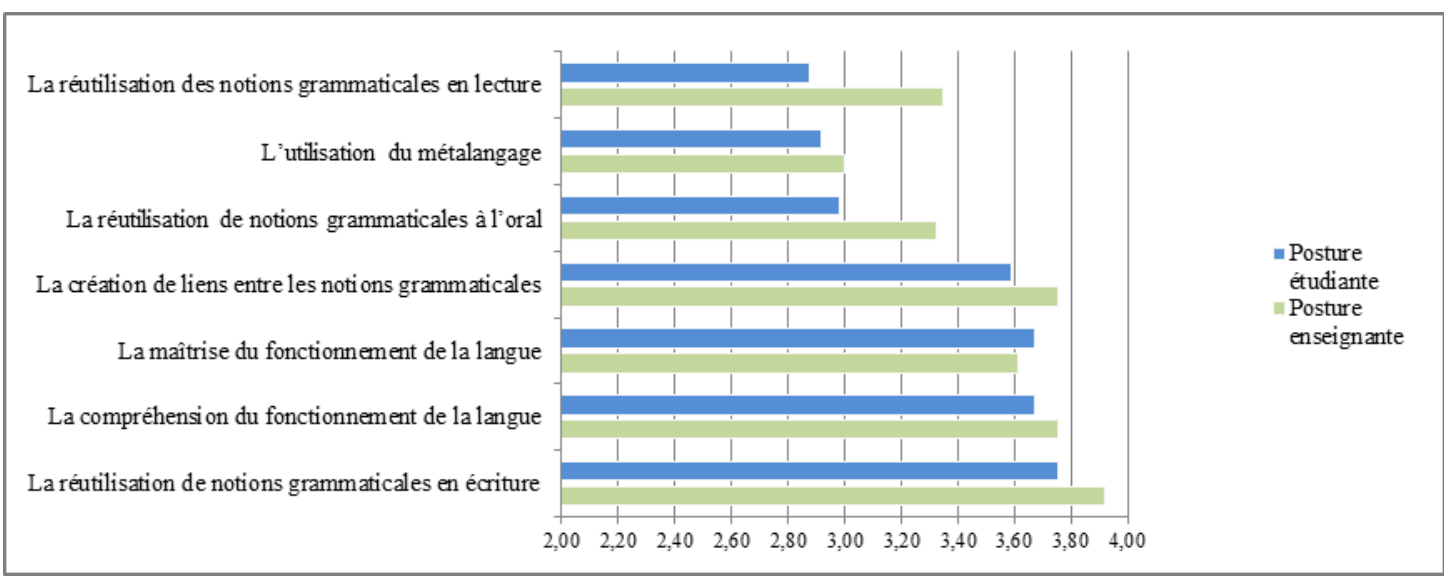

Nous relevons une différence significative (test de rangs signés de Wilcoxon, $p=0,001$ ) entre l'importance donnée à la réutilisation des notions grammaticales en lecture en tant qu'enseignant (moyenne $=3,35 / 4)$ et celle donnée lorsque cet aspect est considéré en tant qu'étudiant (moyenne $=2,88 / 4)$. Il en est de même pour la réutilisation de notions grammaticales en écriture (moyennes $=3,92 / 4$ vs 3,76/4) et à l'oral (moyennes $=3,33 / 4$ vs 2,98/4) (test de rangs signés de Wilcoxon, $\mathrm{p}<0,01)$. Dans l'ensemble, l'importance qu'ont accordée les étudiants aux différents aspects de la grammaire pour l'apprentissage de leurs futurs élèves est plus prononcée que l'importance accordée à ces mêmes aspects en tant qu'étudiant (3,35/4 étudiant vs 3,53/4 enseignant, test de rangs signés de Wilcoxon, $\mathrm{p}=0,007)$. De plus, les tests du Khi-carré confirment qu'aucune réponse n'est distribuée aléatoirement (Khi-2 $>9,53$ pour tous les items, $\mathrm{p}<0,02$ ), que ce soit en tant qu'étudiant ou en tant qu'enseignant.

Nous avons voulu en savoir davantage sur les activités d'enseignement que les futurs enseignants comptaient mettre en place quand ils seraient titulaires de classes de français grâce à la question suivante : En tant que futur enseignant, quelle importance accordez-vous aux activités suivantes d'enseignement de la grammaire (question 18)? Le graphique 6 présente le degré d'importance (très important, plutôt important, peu important, pas important, activité inconnue) que les futurs enseignants accordent à 17 activités d'enseignement.

Graphique 6

Importance accordée par de futurs enseignants à certaines activités d'enseignement 


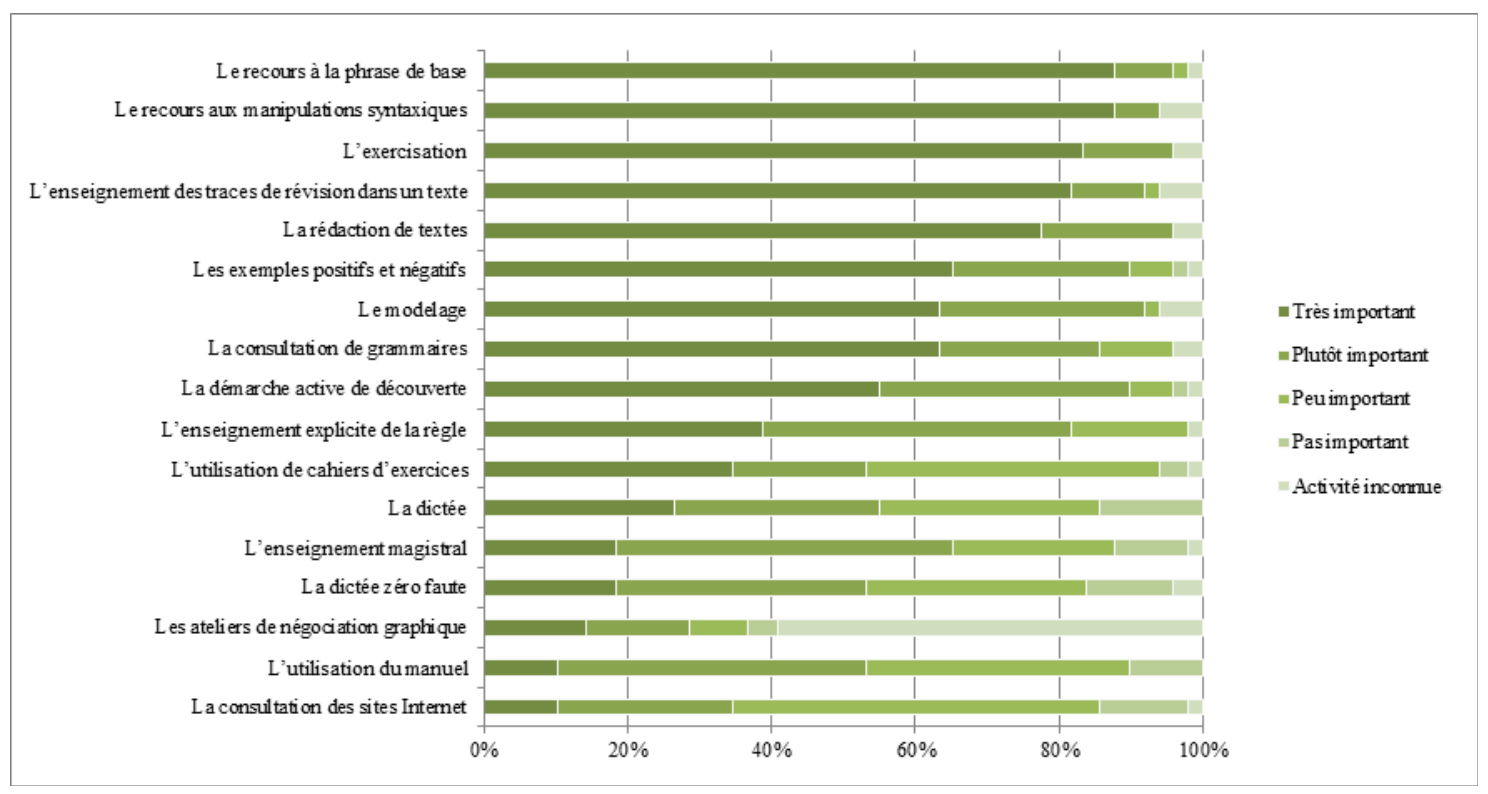

Le graphique 6 montre que $87,7 \%$ des futurs enseignants croient que le recours à la phrase de base et aux manipulations syntaxiques sont des activités d'enseignement très importantes. La rédaction de textes et l'exercisation sont considérées plutôt ou très importantes par environ $96 \%$ des étudiants. L'importance attachée à certaines activités est plus divisée; la dictée $(44,9 \%)$, la dictée zéro faute ${ }^{16}(42,9 \%)$, l'utilisation du manuel $(46,9 \%)$ et l'utilisation de cahiers d'exercices $(44,9 \%)$ sont jugés pas ou peu importantes par près de la moitié des répondants. Seul 9 étudiants ont jugé très important l'enseignement magistral $(18,4 \%)$ et 5 l'utilisation du manuel $(10,2 \%)^{17}$. Près de 6 étudiants sur $10(59,2 \%)$ ont dit ne pas connaitre les ateliers de négociation graphique. Enfin, malgré le fait que 7 étudiants sur 10 soit âgés entre 18 à 25 ans, $63,6 \%$ accordent pas ou peu d'importance à la consultation des sites Internet comme activité d'enseignement de la grammaire. La distribution des items n'est pas aléatoire (Khi-2 > 15,796, p <0,01), excepté pour l'importance accordée à la dictée (Khi-2 =3,163, p =0,367).

Une question portait sur des «actions » que les futurs enseignants jugent importantes d'accomplir pour soutenir leurs futurs élèves dans leur apprentissage de la grammaire. À la question En tant que futur enseignant, quelle importance accordez-vous aux actions suivantes dans l'apprentissage de la grammaire pour vos élèves (question 23)?, les futurs enseignants devaient déterminer le degré d'importance (très important, plutôt important, peu important, pas important) qu'ils accordent à 14 «actions ». Le graphique 7 montre notamment que les étudiants croient unanimement qu'il est très important ou plutôt important de poser des questions aux élèves et de faire des liens entre la grammaire et l'écriture.

Graphique 7

Importance accordée par de futurs enseignants à des « actions » à accomplir pour l'apprentissage de leurs élèves en grammaire 


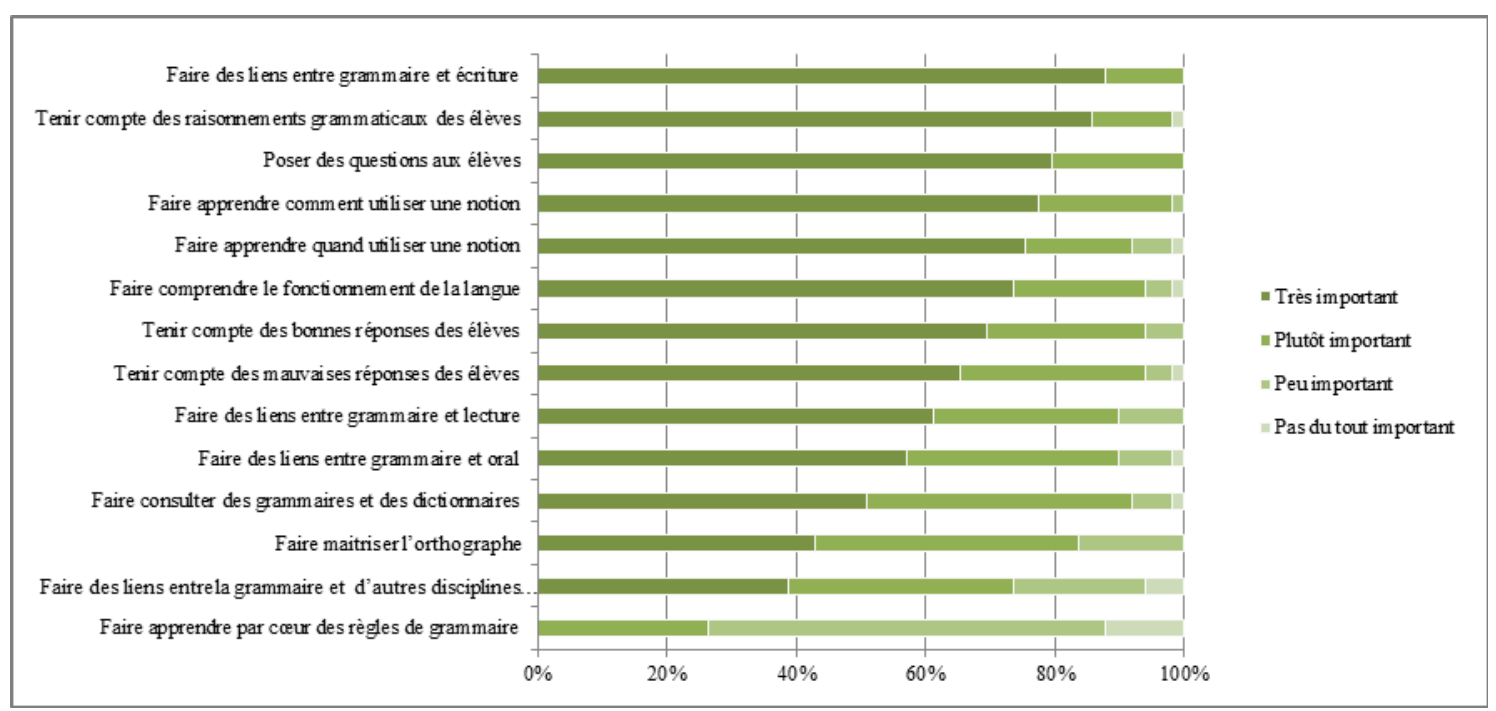

Tenir compte des raisonnements grammaticaux des élèves et leur faire apprendre comment utiliser une notion sont deux «actions » jugées très ou plutôt importantes par $98 \%$ des répondants. Bien que faire maitriser l'orthographe et faire des liens entre la grammaire et d'autres disciplines ont aussi obtenu de bons degrés d'adhésion (très important et plutôt important, 83,7\% et 73,5\%), seul 4 étudiants sur 10 ont qualifié ces actions de très importantes. Résultat similaire pour faire consulter des grammaires et des dictionnaires, «action » jugée très importante pour 5 étudiants sur 10. Aucun étudiant n'a répondu qu'il est très important de faire apprendre des règles de grammaire; 73,5\% ont jugé qu'il s'agissait d'une « action » peu ou pas du tout importante pour l'enseignement de la grammaire.

Enfin, plus largement, nous avons inclus une question portant sur le rôle de la grammaire dans le développement des compétences langagières de l'élève. Les étudiants ont complété l'énoncé Pour développer les compétences langagières de vos élèves, la grammaire est... (question 29) en sélectionnant un des quatre degrés proposés (indispensable, très utile, peu utile, inutile). La quasi-totalité d'entre eux ont choisi indispensable $(51 \%)$ et très utile $(44,9 \%)$. Seul 2 étudiants sur 49 croient que la grammaire est peu utile $(4,1 \%)$ pour développer les compétences langagières des élèves, et aucun croit qu'elle est inutile.

Nous terminerons la présentation des résultats par deux questions posées aux participants. La première : Globalement, en tant qu'étudiant universitaire, vous estimez que vos connaissances en grammaire sont... (question 13). Parmi les 49 répondants, 9 affirment qu'elles sont excellentes (18,4\%), 30 satisfaisantes $(61,2 \%), 9$ plus ou moins satisfaisantes $(18,4 \%)$ et 1 insatisfaisantes $(2 \%)$. Il semble donc que 8 étudiants sur $10(79,6 \%)$ estiment que leurs connaissances grammaticales sont au moins satisfaisantes, de même que pour leur capacité à enseigner la grammaire. En effet, à la question En tant que futurs enseignants, vous estimez que vos capacités à enseigner la grammaire sont... (question 26), 6 affirment qu'elles sont excellentes $(12,2 \%), 33$ satisfaisantes $(67,3 \%), 10$ plus ou moins satisfaisantes $(20,4 \%)$ et 0 insatisfaisantes. Pourtant, les résultats préliminaires de notre recherche en cours suggèrent que les savoirs en grammaire et en didactique de la grammaire des futurs enseignants sont fragiles, en contexte de stage du moins (Gauvin et collaborateurs, soumis).

\section{Discussion : dimensions du rapport à la grammaire et sa didactique de futurs enseignants}

Les résultats nous permettent de proposer une conceptualisation, préliminaire, du rapport à la grammaire et à la didactique de la grammaire de futurs enseignants. Évidemment, la plus grande prudence est requise 
dans l'interprétation: des analyses supplémentaires sur un plus grand échantillon sont encore requises. Toutefois, en l'état actuel d'avancement de notre réflexion, nous voyons émerger deux dimensions du rapport à la grammaire et à sa didactique. Nous les appelons, pour l'instant, dimension subjective et dimension cognitive.

Les résultats suggèrent que les futurs enseignants de français du secondaire abordent la grammaire et son enseignement sous l'angle d'une dimension subjective, de part les valeurs et les sentiments qu'ils entretiennent à leur égard. La grammaire est envisagée comme un objet d'apprentissage auquel les étudiants attribuent une valeur ou des sentiments à la fois positifs et négatifs (question 4). À la question la grammaire, c'est..., les futurs enseignants répondent plus spontanément en évoquant des valeurs ou des sentiments qu'en proposant une définition de l'objet (question 4). Ils éprouvent des sentiments généralement positifs envers leur propre apprentissage de la grammaire (question 7), sentiments qui sont encore plus favorables lorsqu'ils envisagent leur futur enseignement de la grammaire (question 17), comme s'ils entretenaient une vision romantique de son enseignement ${ }^{18}$.

Les résultats suggèrent également que les futurs enseignants de français abordent la grammaire et son enseignement sous l'angle d'une dimension cognitive, de part les manières dont ils se représentent les objets grammaticaux et didactiques et leurs fonctions. Les deux paragraphes suivants présentent quelques faits saillants.

Sur le plan des objets grammaticaux, notons d'abord que les futurs enseignants sont plutôt confiants en leurs connaissances grammaticales (question 13) et en leur capacité à les enseigner (question 26). Plusieurs ont une vision de la grammaire qui se limite principalement à des objets spécifiques (comme la syntaxe ou l'orthographe grammaticale), alors que la vision de certains s'élargit à l'idée de système ou d'ensemble de règles (question 4). La maitrise du métalangage ne semble pas prioritaire pour eux, principalement en situation d'enseignement (questions 11 et 24). En ce qui concerne les objets didactiques, la dictée semble être controversée, alors que la rédaction de textes et l'exercisation font davantage consensus (question 18). Bonne nouvelle : tenir compte des raisonnements grammaticaux des élèves et enseigner à utiliser une notion semblent être des savoirs didactiques largement partagés (question 23).

Sur le plan des fonctions accordées à la grammaire et à son enseignement, la fonction «maitrise du fonctionnement de la langue » accordée à la grammaire et à son enseignement semble dominante, en contraste avec la fonction «compréhension du fonctionnement de la langue » : ce constat devra toutefois être précisé et nuancé dans nos travaux futurs (questions 4, 5, 9, 10,11, 20, 21, 24). Si l'utilité de la grammaire en écriture semble être une évidence autant pour l'apprentissage que l'enseignement de la grammaire, elle ne semble pas l'être pour la communication orale et, surtout, pour la lecture (questions 4, $5,9,11,20,23,24)$. Enfin, presque tous les futurs enseignants s'entendent pour dire que la grammaire est très utile, voire indispensable, pour développer les compétences langagières des élèves.

Le portrait que nous venons d'esquisser semble soutenir la définition que nous proposions (section 2.3) du rapport à la grammaire et à la didactique de la grammaire, soit :

La disposition d'un futur enseignant à l'égard des objets « grammaire » et « didactique de la grammaire », de même qu'à l'égard de la mise en œuvre pratique de ces objets dans sa vie personnelle (d'apprenant) et sa vie professionnelle (d'enseignant).

En ce sens, nous pensons avoir contribué, quoique modestement, à la conceptualisation de cette notion.

\section{Conclusion}

D'entrée de jeu, nous avons indiqué que l'objectif de cet article était de discuter en quoi la notion de rapport à peut être importée aux objets " grammaire » et " didactique de la grammaire », deux objets de savoir que doivent s'approprier les futurs enseignants de français au secondaire. Si la définition proposée (section 2) et les résultats présentés (section 4) nous permettent de croire qu'il est possible d'envisager que le rapport à la grammaire et le rapport à la didactique de la grammaire sont des notions concevables 
et productives pour la didactique de la grammaire, ils nous ont assurément permis de lancer le débat dans l'arène des didacticiens de la langue! Évidemment, la conceptualisation que nous proposons est encore embryonnaire et mérite d'être débattue (section 5), mais elle nous apparait prometteuse. À la lumière des quelques résultats préliminaires présentés, il est indiscutable que les recherches des prochaines années doivent se pencher davantage sur le rapport à la grammaire et le rapport à la didactique de la grammaire des enseignants, qu'ils soient en formation, débutants ou chevronnés; en tant que formateurs d'enseignants de français du secondaire, mais également du primaire, nous pouvons intervenir sur ces rapport à. Mais avant, il faut évidemment mieux les connaitre.

Montréal, 15 mars 2014

\section{Références bibliographiques}

Anctil, D. (2010). L'erreur lexicale au secondaire : analyse d'erreurs lexicales d'élèves de 3e secondaire et description du rapport à l'erreur lexicale d'enseignants de français. Thèse de doctorat: Université de Montréal.

Barré-De Miniac, C. (2000). Le rapport à l'écriture, aspects théoriques et didactiques. Paris : Septentrion.

Barré-De Miniac, C. (2002a). Le rapport à l'écriture. Une notion à plusieurs dimensions. Pratiques, 113-114, 29-40.

Barré-De Miniac, C. (2002b). Du rapport à l'écriture de l'élève à celui de l'enseignant. Revue Éduquer, 2, 3-10.

Barré-De Miniac, C. (2008). Le rapport à l'écriture : une notion à valeur euristique. Dans S.-G. Chartrand \& C. Blaser (Dirs.), Le rapport à l'écrit : un outil pour enseigner de l'école à l'université (pp. 11-23). Namur: Presses universitaires de Namur.

Charlot, B. (1997). Du rapport au savoir, éléments pour une théorie. Paris : Anthropos.

Chartrand, S.-G., \& Blaser, C. (2008). Du rapport à l'écriture au concept didactique de capacités langagières : apports et limites de la notion de rapport à l'écrit. Dans S.-G. Chartrand \& C. Blaser (dirs.), Le rapport à l'écrit : un outil pour enseigner de l'école à l'université (pp. 107-127). Namur : Presses universitaires de Namur.

Chartrand, S.-G., \& Prince, M. (2009). La dimension affective du rapport à l'écrit d'élèves québécois. Revue canadienne de l'éducation, 32(2), 317-343.

Dionne, A.-M. (2010). Le rapport à la lecture et les compétences en écriture des futurs enseignants : enjeux déterminants pour favoriserle goût de lire chez les élèves. Revue des sciences de l'éducation de McGill, 45(3), 409428.

Émery-Bruneau, J. (2010). Le rapport à la lecture littéraire. Des pratiques et des conceptions de sujets-lecteurs en formation à l'enseignement du français à des intentions didactiques. Thèse de doctorat: Université Laval.

Émery-Bruneau, J. (2011). La dimension sociale du rapport à la lecture littéraire d'étudiants en enseignement : un indice du développement de l'identité professionnelle. Revue canadienne de l'éducation, 34(2), 34-52.

Falardeau, É., \& Simard, D. (2004). Le rapport à la culture des enseignants de français et son rôle dans l'articulation de la culture avec les contenus disciplinaires. Actes du $9^{\mathrm{e}}$ colloque de l'AIRDF, Québec.

Falardeau, É., \& Simard, D. (2009). La grammaire en classe de français au secondaire : affectivité des élèves et pratiques enseignantes. Dans J. Dolz \& C. Simard (dirs.), Pratiques d'enseignement grammatical. Points de vue de l'enseignant et de l'élève (pp. 233-254). Québec : Presses de l'Université Laval.

Gauvin, I., Boivin, M.-C., Duchesne, J., Lefrançois, P., Ouellet, C., Pinsonneault, R., \& Simard, D. (soumis). Les savoirs en grammaire et en didactique de la grammaire de futurs enseignants: une porte d'entrée pour réexaminer la formation à l'enseignement. Colloque du REF, Genève.

Nadeau, M., \& Fisher, C. (2011). Les connaissances implicites et explicites en grammaire: quelle importance pour l'enseignement? Quelles conséquences? Bellaterra Journal of Teaching \& Learning Language \& Literature, 4(4), 131. 
Reuter, Y., Cohen-Azria, C., Daunay, B., Delcambre, I., \& Lahanier-Reuter, D. (2007). Dictionnaire des concepts fondamentaux des didactiques. Bruxelles : Éditions De Boeck.

\footnotetext{
${ }^{1}$ Symposium organisé par Suzanne-G. Chartrand, Université Laval, et Roxanne Gagnon, Université de Genève, dans le cadre du colloque du Réseau international de recherche en Éducation et en Formation (REF), Genève, septembre 2013.

${ }^{2}$ Combettes et Largarde (1982) ont été menés dans un contexte français, il y plus 30 ans.

${ }^{3}$ Recherche Savoirs en grammaire et en didactique de la grammaire chez des étudiants en enseignement du français au secondaire. Subvention du Fonds de recherches du Québéc, 2012-2015, 135 999\$. Isabelle Gauvin, professeure à l'Université du Québec à Montréal, est chercheuse principale. Reine Pinsonneault, Daphnée Simard et Chantal Ouellet de l'Université du Québec à Montréal ainsi que Marie-Claude Boivin, Pascale Lefrançois, Mireille Tremblay et Michel Lepage de l'Université de Montréal sont cochercheurs. Profonds remerciements à tous les assistants de recherche, plus précisément à Renée Lemay, Sylvie Marcotte, Marie-Claude Paventi et Karine Villeneuve qui ont travaillé d'arrache-pied pour rendre disponibles les résultats présentés dans cet article. Chaleureux remerciements à Jill Vandermeerschen, statisticienne au Département de mathématiques de Université du Québec à Montréal, pour ses précieux conseils.

${ }^{4}$ La recherche a pour objectif général de documenter les savoirs en grammaire et en didactique de la grammaire de futurs enseignants et de stagiaires (Gauvin, Boivin, Duchesne, Lefrançois, Ouellet, Pinsonneault et Simard, 2013).

${ }^{5}$ Nous reviendrons sur cet aspect fondamental en 2.4, Pourquoi s'intéresser au rapport à la grammaire et à sa didactique.

${ }^{6}$ Nous reconnaissons la pertinence de la remarque d'un évaluateur anonyme qui regrette qu'aucun passage de la contribution ou item du questionnaire ne fasse explicitement mention du « rapport à la langue » chez les enquêtés. En effet, notre outil de collecte ne prévoit pas interroger les futurs enseignants sur leur rapport à langue. Toutefois, les résultats obtenus nous permettront sans doute d'explorer les liens possibles entre le rapport à la grammaire et son enseignement, objets de notre étude, et, plus largement, leur rapport à la langue.

${ }^{7}$ Ce qui inclut non seulement l'écriture, mais également la lecture.

${ }^{8}$ Rappelons la définition présentée plus haut : le rapport à concerne « la disposition (d'une) personne à l'égard d'un objet » et " à l'égard de la mise en cuvre pratique de cet objet dans la vie personnelle, culturelle, sociale et professionnelle » (Barré-De Miniac, 2000, p.13).

${ }^{9}$ Fait étonnant : les chercheurs ont obtenu ce résultat alors qu'aucune question ne portait sur la grammaire et son enseignement.

${ }^{10}$ Au moment d'écrire ces lignes, l'échantillon n'est pas complet, la collecte des données étant toujours en cours auprès d'une cinquante d'étudiants supplémentaires. Ces nouvelles données sont susceptibles de modifier quelque peu les résultats présentés ici.

${ }^{11}$ Des résultats issus des réponses à ces questions ont déjà été présentés (Gauvin et collaborateurs, soumis 2013).

${ }^{12}$ Cette question est inspirée des travaux de Christiane Blaser, professeure à l’Université de Sherbrooke.

${ }^{13}$ Afin de distinguer les deux postures des répondants, la couleur bleue est utilisée dans les tableaux et les graphiques pour la posture « étudiant », la couleur verte pour la posture « enseignant ».

${ }^{14}$ Cette différence n'est problablement pas significative ; d'autres analyses sont à venir.

15 À la question 24, la formulation de 3 activités diffère légèrement : «bien vous exprimer », « vous aider à lire » et « vous aider à communiquer » deviennent « bien s'exprimer », « mieux lire », « mieux communiquer ».

${ }^{16}$ Pour en connaître davantage sur cette activité, voir Nadeau et Fisher, 2011.

${ }^{17}$ Dans notre recherche, le questionnaire n'est pas le seul outil de collecte de données. Parmi l'ensemble des répondants, 12 étudiants ont été filmés pendant toute la durée de leur troisième stage de formation en milieu de pratique. L'analyse préliminaire des données des films de classe laisse entrevoir une différence entre les activités jugées importantes et celles effectives lors de l'enseignement.

${ }^{18}$ Nous remercions Karine Villeneuve, assistante de recherche et romantique à ses heures, qui a fait cette poétique proposition.
} 